PALEO

Revue d'archéologie préhistorique

19 | 2007

Spécial table ronde ( 1 ère partie) : Le Gravettien : entités régionales d'une paléoculture européenne, Les Eyzies, juillet 2004

\title{
Le Gravettien de la côte méditerranéenne ibérique
}

Gravettian in the iberian mediterranean coast

Josep M. Fullola, Dídac Roman, Narcis Soler et Valentin Villaverde

\section{OpenEdition}

Journals

Édition électronique

URL : https://journals.openedition.org/paleo/523

DOI : $10.4000 /$ paleo.523

ISSN : 2101-0420

Éditeur

SAMRA

Édition imprimée

Date de publication : 30 décembre 2007

Pagination : 73-88

ISSN : $1145-3370$

Référence électronique

Josep M. Fullola, Dídac Roman, Narcis Soler et Valentin Villaverde, «Le Gravettien de la côte

méditerranéenne ibérique », PALEO [En ligne], 19 | 2007, mis en ligne le 23 avril 2009, consulté le 29

juin 2021. URL : http://journals.openedition.org/paleo/523; DOI : https://doi.org/10.4000/paleo.523

\section{(C) $\oplus \Theta \Theta$}

PALEO est mis à disposition selon les termes de la licence Creative Commons Attribution - Pas d'Utilisation Commerciale - Pas de Modification 4.0 International. 


\title{
LE GRAVETTIEN DE LA CÔTE MÉDITERRANÉENNE IBÉRIQUE \\ (1)
}

\author{
Josep M. FULLOLA (2), Dídac ROMAN ${ }^{(3)}$, \\ Narcis SOLER ${ }^{(4)}$ et Valentin VILLAVERDE ${ }^{(5)}$
}

\begin{abstract}
Résumé : Même si les données disponibles pour le Gravettien méditerranéen ibérique sont réduites et dans des contextes stratigraphiques mal définis, nous présentons dans ce travail les révisions de quelques sites majeurs (Reclau Viver, l'Arbreda, Parpalló, Malladetes, Beneito et Cendres ainsi que de quelques autres gisements. La vision globale du Gravettien méditerranéen ibérique nous montre, dans la période ancienne, une faible présence mais une industrie lithique et osseuse très classique est observée dans les phases moyenne et finale de la période.
\end{abstract}

Mots-clés : Paléolithique supérieur, Gravettien, Péninsule ibérique, Méditeranée.

Abstract: Gravettian in the iberian mediterranean coast Even if data coming from Gravettian of the iberian mediterranean coast are few, and only some of them in consistent stratigraphic contexts, we review in this paper some of the main sites (Reclau Viver, L'Arbreda, Parpalló, Malladetes, Beneito and Cendres); all them, with other minor sites, can give us a global perspective of iberian mediterranean Gravettian, with little presence in the early moments, but with classical lithic and bone industries in the middle and late periods of this phase.

Key words: Upper Palaeolithic, Gravettian, Iberia, Mediterranean.

Depuis les années 1950, le Gravettien est considéré comme une étape importante dans la dynamique culturelle du Paléolithique supérieur de la région méditerranéenne ibérique. Jordà (1955) comme Pericot (1963) associent à cette phase la consolidation du peuplement de cette région et la mise en place de caractères culturels qui vont perdurer du Solutréen jusqu'à l'Epipaléolithique. Depuis ces travaux, les gisements présentant des matériaux de cette étape ont intégré et précisé la vision antérieure, surtout à partir des travaux de Fortea (1973) puis Fortea et Jordà (1976) qui ont permis de déterminer la position chronologique des industries épigravettiennes, se détachant du Paléolithique supérieur. Nous évoquons, dans ce travail, un panorama du Gravettien méditerranéen ibérique (fig. 1) en intégrant les données nouvelles de gisements de Catalogne et du Pays valencien. Nous aborderons également la caractérisation et les problèmes de l'origine du Gravettien régional et de ses relations avec le Solutréen.

\section{LE GRAVETTIEN EN CATALOGNE}

Le Gravettien n'est pas très présent en Catalogne. On le trouve dans les gisements de Serinyà et dans d'autres sites isolés. Les niveaux gravettiens sont placés au-dessus de l'Aurignacien et sous le Solutréen dans trois grottes stratifiées de Serinyà (Girona): le Reclau Viver, l'Arbreda, et Davant Pau.

Dans ces trois gisements majeurs mais également à Mollet III, situés tous les quatre dans la vallée de Serinyà, le Gravettien présente une certaine unité. Celle-ci peut être étendue à un autre gisement stratifié, l'abri Romaní (Capellades) où il y a, dans la couche 2 , quelques outils très comparables à ceux de la vallée de Serinyà.

Plus difficiles à interpréter, d'autres gisements peuvent être assimilés au Gravettien par leur industrie ou leur datation :

(1) Ce travail a été fait avec l'aide des projets BHA2002-02342 et HAR2008-00103 et HUM 2007-63435 du Ministère espagnol de l'Education et des Sciences et dans le cadre du Groupe de Recherche de Qualité du SERP SGR2005-00299 de la Generalitat de Catalogne.

(2) Université de Barcelone - Département de Préhistoire, Faculté de Géographie et Histoire, Rue Montalegre, 6; E-08001, Barcelone, Espagne et Directeur du SERP (Séminaire d'Études et Recherches Préhistoriques) - fullola@ub.edu (3) Université de Valence, Département de Préhistoire, Faculté de Géographie et Histoire, Avenida Blasco Ibáñez, 36; E46071, Valence, Espagne

(4) Université de Gérone, Département Préhistoire, - Plaça Ferrater i Mora, 1, 17071, Gerone, Espagne - narcis.soler@udg.edu (5) Université de Valence, Département Préhistoire, Faculté de Géographie et Histoire, Avenida Blasco Ibáñez, 36; E-46071, Valence, Espagne - valentin.villaverde@uv.es (auteur pour la correspondence). 


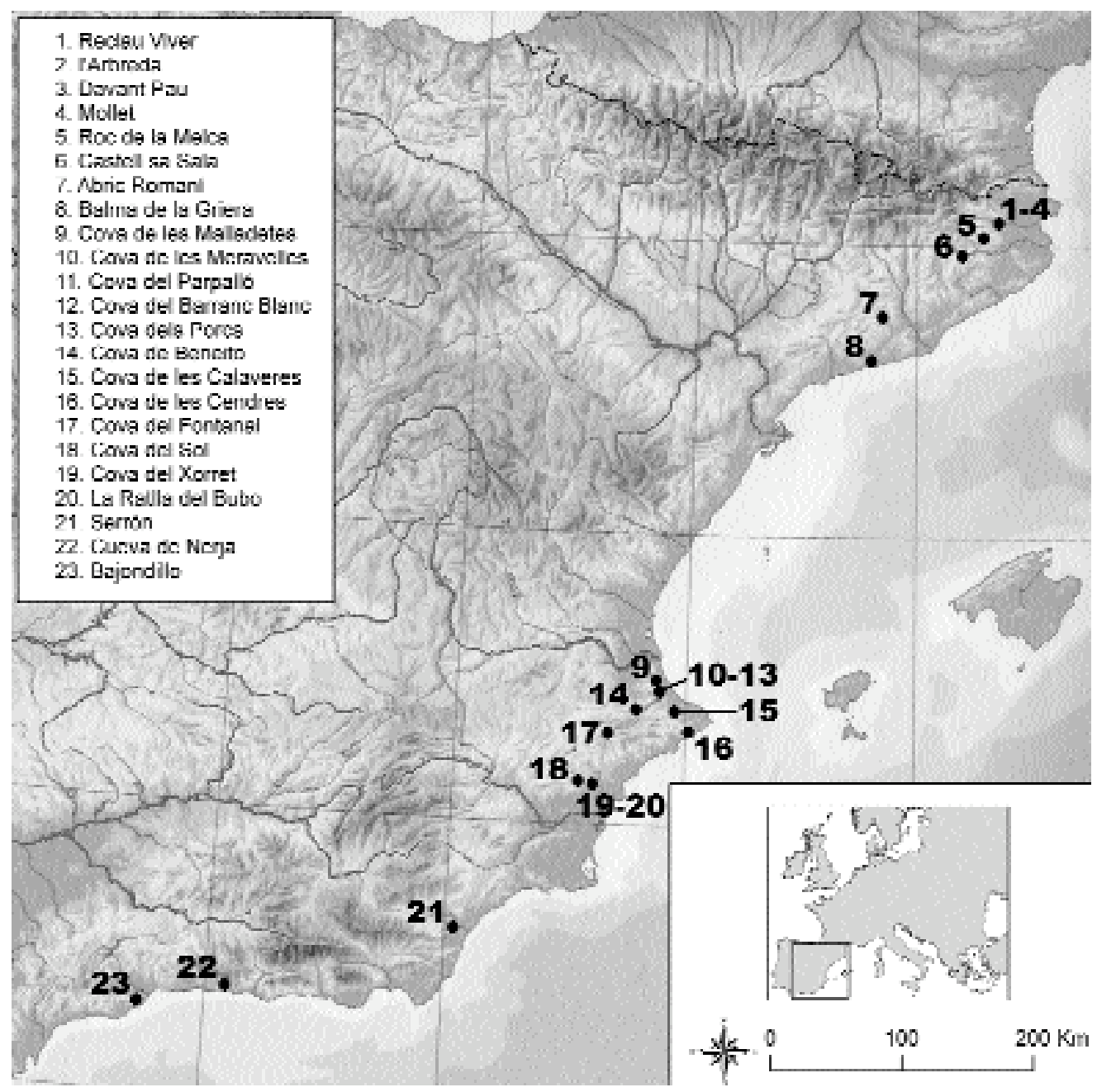

Figure 1 - Carte de localisation des gisements gravettiens de l'aire méditerranéenne ibérique.

Figure 1 - Location map of the gravettian sites in the Iberian mediterranean area.

le Roc de la Melca, Castell Sa Sala, la Griera et quelques trouvailles superficielles (Soler et Maroto 1996) (fig. 2 et 3).

\section{La grotte de Reclau Viver}

Ce gisement catalan présente la séquence la plus importante pour le Gravettien régional. II a été fouillé par J. M. Corominas de 1944 à 1948 (Corominas 1946, 1949). Entre l'Aurignacien (couches A et B) et le Solutréen (couche F), il signala la présence de trois niveaux (C, D et $E$ ).

Le niveau $\mathrm{C}$ est caractérisé par la présence de pointes à dos microlithiques (microgravettes pygmées) et de lamelles à dos. Les outils à retouche abrupte représentent $48 \%$ de l'ensemble retouché (lamelles à dos $32 \%$, pointes à dos 14 $\%)$. Les grattoirs $(14,7 \%)$ sont plus abondants que les burins $(10,3 \%)$; préférentiellement sur troncature. Le fouilleur considérait le niveau C comme Périgordien IV, d' après les classifications de D. Peyrony $(1933,1946)$.

Le niveau $D$ est situé au contact et au-dessus du niveau C. En relation avec ce dernier, il est caractérisé par la diminution de la fréquence de l'outillage microlithique, l'augmentation de la taille des outils et par la présence d'une industrie osseuse plus riche. Les outils à retouche abrupte représentent $23 \%$ (lamelles à dos $11 \%$, pointes à dos $10 \%$, parmi lesquelles des pointes de la Gravette bien typiques). Les grattoirs sont toujours abondants (14\%) comme les burins (13,4\%), souvent sur troncature.

L'industrie osseuse est caractérisée par les sagaies à base oblique. Pour cette raison, Corominas $(1946,1949)$ attribua ce niveau à l'Aurignacien $\mathrm{V}$ de Peyrony. En ce qui concerne la parure, elle est très abondante avec plusieurs canines de camivores percées et aussi quelques têtes de fémurs de cervidés percées.

Sur le niveau $\mathrm{D}$, on trouve le niveau $\mathrm{E}$ considéré comme Protosolutréen ou Solutréen qui possède encore des gravettes typiques parmi les pointes à dos, des outils qui existent aussi dans le niveau F, nettement Solutréen.

\section{La grotte de l'Arbreda}

Cet autre gisement qui présente le Gravettien stratifié entre l'Aurignacien et le Solutréen, est la plus grande des grottes du Reclau, avec une stratigraphie considérable, de plus de $10 \mathrm{~m}$ d'épaisseur, qui comprend plusieurs niveaux du Paléolithique supérieur et du Paléolithique moyen. 

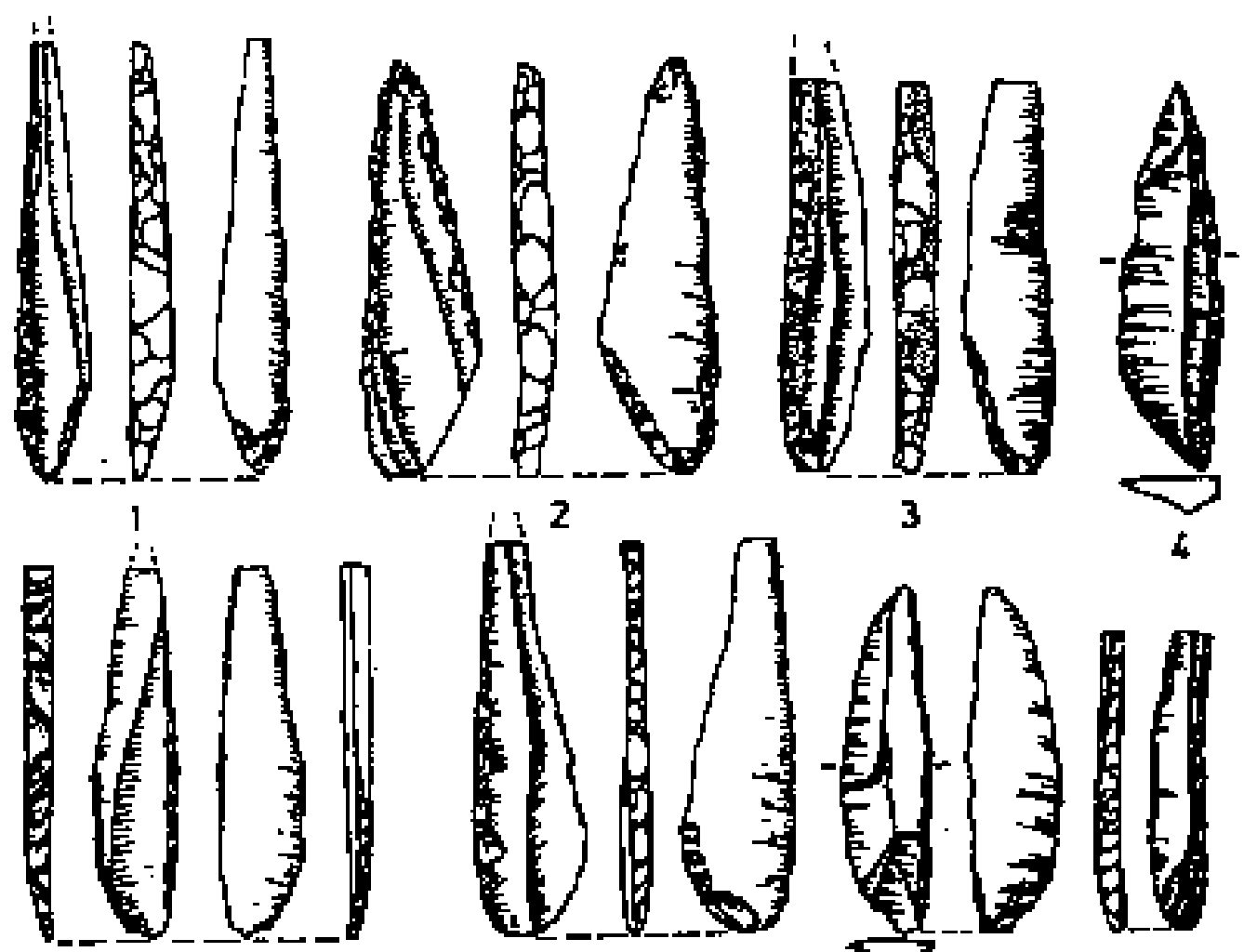

5
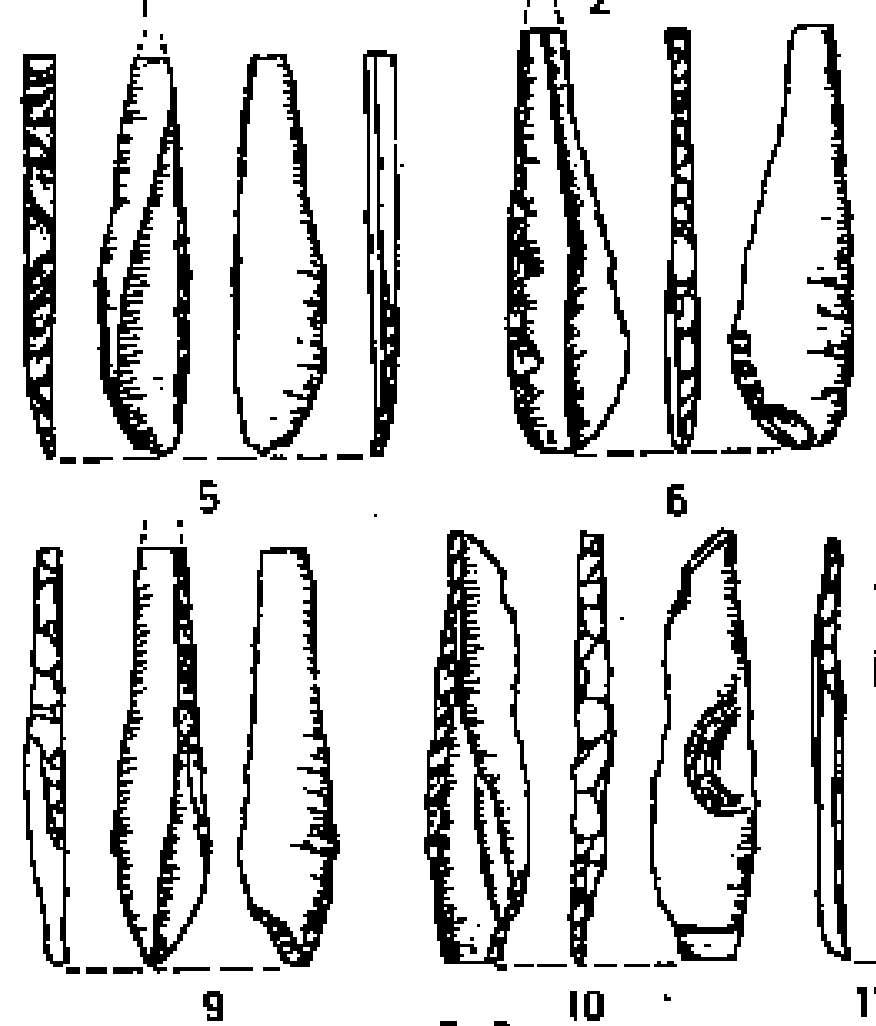

5
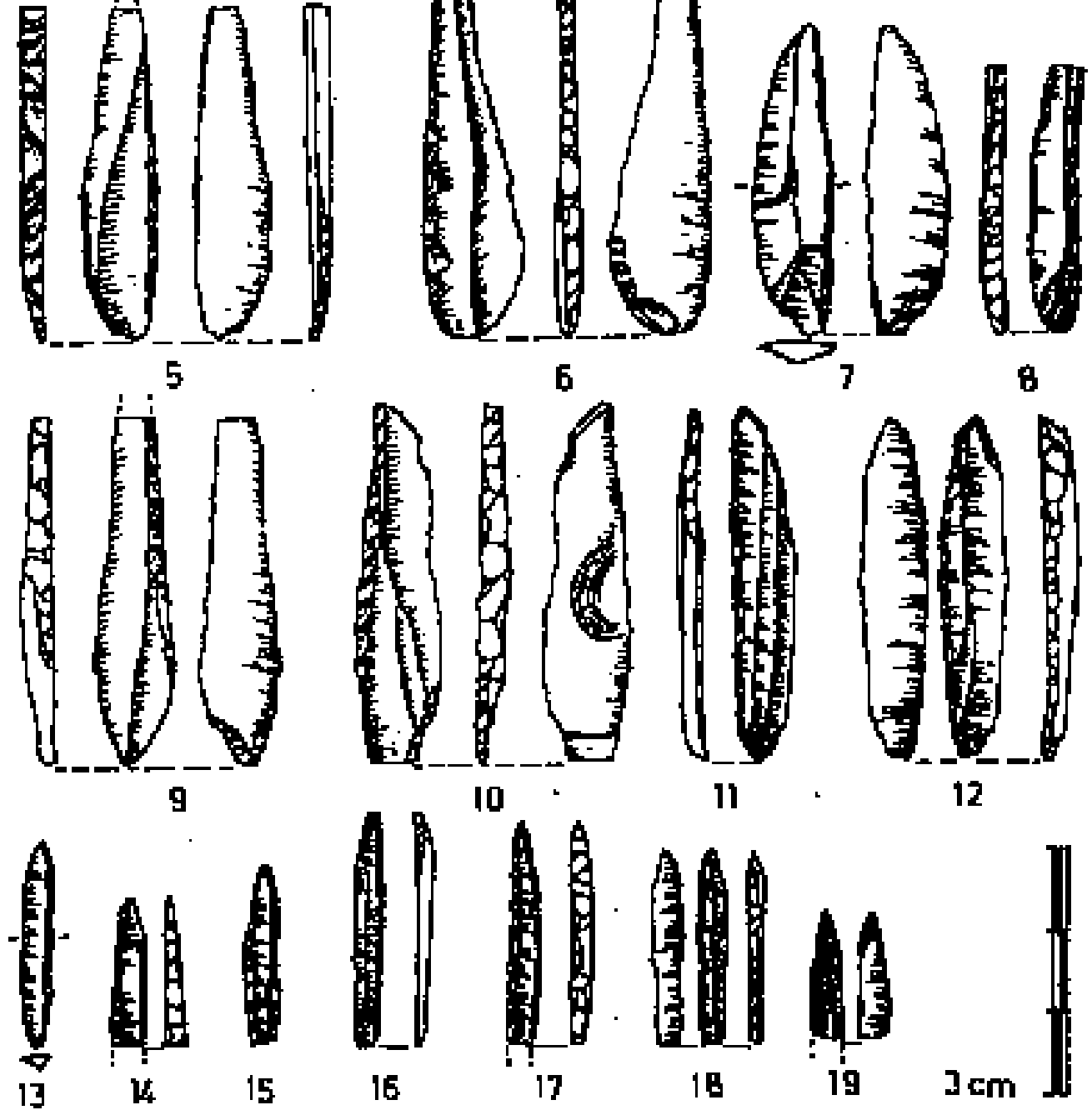

12

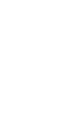

Figure 2 - Pointes et lames à dos gravettiennes des sites de la région du Reclau de Serinyà. 1, 2, 8 : Reclau Viver niveau D; 3 , 4, 11-19 : Reclau Viver niveau C; 7 : Reclau Viver vestibulo; 5 : Davant Pau; 6, 9, 10 : Arbreda.

Figure 2 - Gravettian backed points and bladelets of the sites of the Reclau are in Serinyà. 1, 2, 8 : Reclau Viver level D; 3, 4, 11-19: Reclau Viver level C; 7 : Reclau Viver oputside area; 5 : Davant Pau; 6, 9, 10 : Arbreda. 

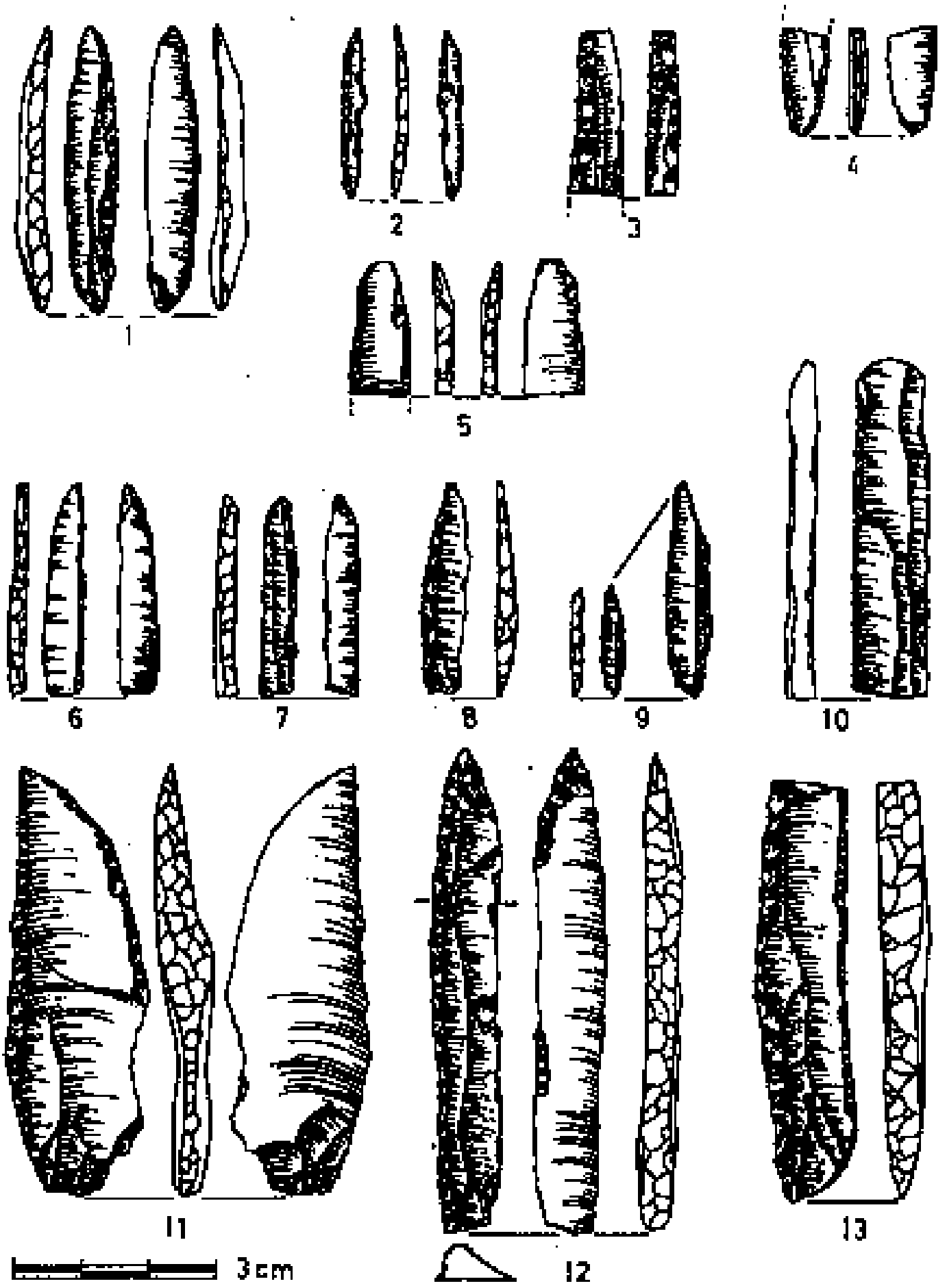

Figure 3 - Pointes et lames à dos gravettiennes de Catalogne. 1-5 : Abri Romaní; 6-10 : Castell Sa Sala; 11-13 : Reclau Viver niveaux $D$ et $E$.

Figure 3 - Gravettian backed points and bladelets of Catalonia. 1-5 : Abri Romani; 6-10 : Castell Sa Sala; 11-13 : Reclau Viver levels $D$ and $E$. 
Au-dessus de l'Aurignacien évolué du niveau $\mathrm{G}$, daté de 28000 BP (Sacchi et al. 1996), et sous le Solutréen, il y a au moins deux niveaux gravettiens, $E$ et $F$, mais seul le premier est significatif par le nombre d'objets qu'il contient (Soler et Maroto 1987a ; 1987b).

Le niveau $\mathrm{F}$ est très pauvre. Parmi l'industrie lithique, il y a 34 outils, dont sept grattoirs, cinq burins, cinq pointes à dos - avec trois gravettes typiques, huit fragments de pièces à dos et sept racloirs. II y a aussi un poinçon en os et une canine de cerf percée.

Le niveau $E$ a une datation sur charbon de $20130 \pm 220$ BP (GIF-6420) (Delibrias et al. 1987). Nous ferons la description du Gravettien de l'Arbreda à partir de ce niveau.

Les proportions de matières premières de l'ensemble de l'industrie lithique sont de 96,6\% pour le silex et de 3,4\% pour le reste : quartz, quartzite, cornéenne, schiste, roches filoniennes. Ainsi, la proportion de roches locales $(3,4 \%)$ est très faible, ce qui différencie ce niveau des autres niveaux du Paléolithique supérieur des grottes du Reclau, où elles sont plus abondantes. Le silex est rare dans la région et des matériaux allochtones ont donc été apportés sur le site pour le débitage.

L'industrie de ce niveau est typiquement gravettienne, caractérisée par l'abondance des pointes à dos. II y a 237 outils retouchés, tous en silex. Les outils à retouche abrupte forment $70,4 \%$ (fragments de pointes ou lamelles à dos indifférenciés $37,1 \%$, pointes à dos $24 \%$, lamelles à dos $8 \%$ ). Parmi les pointes à dos, il y a quelques gravettes typiques et d'abondantes microgravettes ; ces dernières sont souvent extrêmement petites. Les lamelles à dos sont peu standardisées à l'exception d'une lamelle à dos tronquée.

Les grattoirs constituent 9,7\% des outils. II n'y a aucun grattoir caréné. Ce sont des grattoirs simples ou à retouche latérale ; il y un grattoir-burin. Les burins $(5,9 \%)$ sont dièdres dans la moitié des cas, sinon sur troncature retouchée. Les racloirs, atypiques, représentent 5,9\% de l'ensemble. II y a aussi 3,7\% de lames à retouche marg inale ou profonde. Les denticulés constituent $1,6 \%$ et les écaillés $4,2 \%$ de l'outillage.

L'industrie osseuse est très pauvre. II y a seulement un fragment de sagaie, deux fragments de poinçons et une alêne en bois de cerf.

La parure n'est pas très riche. Elle est constituée de quelques canines percées de cerfs et de camivores et de quelques coquillages qui représentent seulement trois espèces de bivalves : Cardium sp., Pecten jacobeus et Pecten maximus, qui ont été déterminées par J. Oller.

La faune des grands et moyens mammifères est bien représentée. Elle est largement dominée par le lapin (Oryctolagus cuniculus) dont une bonne part est d'origine anthropique.

Les grands mammifères, plus rares, sont dominés par le cheval (Equus caballus), suivi de grands bovidés (Bos primige-
nius-Bison priscus) et du cerf (Cervus elaphus). De façon plus sporadique, y sont représentés Equus hydruntinus, Rupricapra rupricapra, Vulpes vulpes et Erinaceus europaeus.

Dans le niveau E, la présence des arbres est faible. Artemisia et les Asteraceae dominent sur les Poaceae ou graminées sylvestres. Le paysage correspondrait à celui d'une steppe froide. À la fin de la période, il y aurait une hausse de l'humidité et de la température (Burjachs 1993 ; Burjachs et Renault-Miskovsky 1992).

L'analyse anthracologique de ce niveau a donné seulement deux taxons : Pinus sylvestris et Betula verrucosa. Malgré la pauvreté de l'échantillon, l'association de ces deux arbres et l'absence d'espèce méditerranéenne indiquent généralement la diminution des températures (Ros 1987).

Parmi les restes de poissons, ont été identifiés: Anguilla anguilla, Salmo trutta fario, Barbus sp., Leuciscus sp., Salmo salar et Salmo sp. (Muñoz et Casadevall 1997)

Les oiseaux sont représentés dans ce niveau seulement par quatre espèces identifiées: Falco subbuteo, Coturnix coturnix, Pyrrhocorax graculus et Corvus monedula (Garcia 1997).

Les insectivores et les chiroptères ont été étudiés par Àngel Galobart, qui a identifié : Erinaceus europaeus, Sorex minutus, Sorex araneus et Crocidura rusula pour les premiers et Myotis myotis et Plecotus austriacus/auricus pour les seconds.

Parmi les rongeurs, Microtus avalis-agrestis est très abondant et la présence de Microtus oeconomus, une espèce froide et continentale, est significative. II y a aussi Eliomys quercinus, Pitymys sp., Arvicola sp. et Apodemus sylvaticus (Alcalde 1987).

Toutes ces analyses semblent nous indiquer l'existence, pour le niveau $E$, d'un climat froid et sec, certainement avec le niveau Solutréen, le plus froid et le plus sec de la séquence du Paléolithique supérieur de l'Arbreda. On l'identifie au Pléniglaciaire de la demière glaciation et on peut penser à un climat rigoureux d'influence continentale, avec des hivers froids et secs et des étés humides.

\section{Grotte de Davant Pau}

Un sondage de J. M. Corominas devant la grotte d'En Pau montra l'existence d'un niveau gravettien, très peu fouillé (Soler 1986). Les travaux ont révélé des pointes à dos, et entre autres, une pointe de la Gravette typique. On y a trouvé aussi un sifflet sur cubitus de cervidé (Soler et García 1995).

\section{Mollet III}

Dans les environs du Reclau, la grotte de Mollet III possède un petit niveau du Paléolithique supérieur qui a donné quelques canines supérieures de cerf perforées avec une belle pointe de la Gravette, qui nous signale simplement la présence du Gravettien dans le gisement (Soler 1986). 


\begin{tabular}{|ll|l|l|l|l|ll|l|} 
& $\begin{array}{l}\text { Malladetes } \\
\mathbf{1}, 6-2,4\end{array}$ & Malladetes & $\begin{array}{l}\text { Bencito } \\
\text { niv. 6 }\end{array}$ & Bencito & Bencito & Parpalló & Barranc & Cendres \\
niv. 7b & $7,25-8$ & Blanc & XV-Xvi \\
\hline R & 35,9 & 35,1 & 18,5 & 21,4 & 18,6 & 55,8 & 27,8 & 2,9 \\
\hline P & - & 0,4 & 1,5 & 1,8 & 1,2 & - & 0,5 & 0,9 \\
B & 5,9 & 7,1 & 23,1 & 15,2 & 12,8 & 3,5 & 12,8 & 7,8 \\
D & 15,7 & 11,2 & 16,9 & 27,7 & 15,1 & 15,1 & 5,9 & 19,6 \\
I & 0,5 & 2,6 & 4,6 & 11,6 & 5,8 & - & 3,7 & 2,9 \\
\hline I'RC & 22,1 & 24,6 & 6,2 & 3,5 & 6,9 & 10,5 & 27,8 & 15,7 \\
\hline E & 2,3 & 2,6 & - & 1,8 & - & - & 2,7 & 11,8 \\
\hline ml & 7,4 & 2,9 & 9,3 & 10,7 & 36,1 & 10,5 & 2,1 & 26,5 \\
\hline
\end{tabular}

Tableau 1 - Principaux indices typologiques du Gravettien valencien. R: Grattoirs; P: Perçoirs; B: Burins; D: Pièces à dos; T: Tronqués; PRC: Pièces à retouches continues ; E: Pièces esquillées; ml: Outillage microlaminaire retouché.

Table 1 - Main typological index of valentian Gravettian. R: End scrapers; P: Becs; B: Burins; D: Backed elements; T: Truncations; PRC: continous retouched elements; E: Flaked elements (esquillées); ml: Microbladelet retouched elements.

L’abri Romaní (Capellades, Barcelone)

Malgré le fait que presque toutes les occupations de ce gisement appartiennent au Moustérien, dans le niveau supérieur, la couche 2 n'a pu être fouillée que par l'inventeur du site et quelques fouilleurs dans la première décennie du $X X^{\ominus}$ siècle. Elle a été considérée à l'époque comme magdalénienne. En réalité, les matériaux sont très comparables à ceux de la région de Serinyà et on peut y voir d'un côté, du Protoaurignacien ou Aurignacien archaïque et de l'autre, du Gravettien (Laplace 1962 ; Soler 1986). Parmi les six pointes à dos, il y a des gravettes et des microgravettes. Les datations sur charbon ou concrétions donnent des âges propres au Moustérien ou au Protoaurignacien très ancien (Bishoff et al. 1994), mais la présence du Gravettien est indubitable. II y a donc eu un mélange dans les collections.

\section{L'abri du Roc de la Melca (Sant Aniol de Finestres, Gérone)}

Ce petit abri contenait un seul niveau archéologique qui a été daté sur os à $20900 \pm 400 \mathrm{BP}$ (MC-2219) (Soler 1980).

L'industrie lithique contient de nombreux racloirs (13\%) et des denticulés (31,6\%). Les outils à retouche abrupte constituent $33,1 \%$ (lamelles à dos $11,3 \%$, pointes à dos $2,5 \%$ ). Les grattoirs représentent $4,6 \%$ et les burins, la plupart sur troncature retouchée, $13,9 \%$ de l'ensemble retouché.

\section{Castell sa Sala (Vilanova de Sau, Barcelone)}

L'abri du Castell sa Sala fournit à des amateurs locaux une industrie abondante du Paléolithique supérieur (Vila 1987). Nous attribuons cette industrie au Gravettien (Soler 1986). Elle est caractérisée par la présence de quelques microgravettes et de nombreux burins.

II y a $34 \%$ de denticulés et $14 \%$ de racloirs, les outils à retouche abrupte forment $20 \%$ (lamelles à dos 10,3\%, pointes à dos 4,1\%), les grattoirs, 8,6 \% et les burins 21,2\% de l'outillage. Ces derniers sont partagés entre burins dièdres et burins sur troncature $(6,8 \%)$. Parmi les premiers, il y en a quelques uns à retouche d'arête. La présence de burins plans comparables aux burins du Raysse et de Gratadis est également significative.

\section{La Balma de la Griera (Calafell, Barcelone)}

L'abri de la Balma de la Griera a livré dans le niveau inférieur une industrie qui n'a pas fourni de pièces typiques. II a été daté en AMS sur os, à $21255 \pm 350$ BP (AA-8649) et attribué au Gravettien. La faune, Cerf et Cheval, est rare et très fragmentée (Fullola et al. 1994 ; Cebrià et al. 2000).

\section{LE GRAVETTIEN EN PAYS VALENCIEN}

Dans la région valencienne, les gisements présentant des niveaux stratigraphiques gravettiens sont au nombre de 

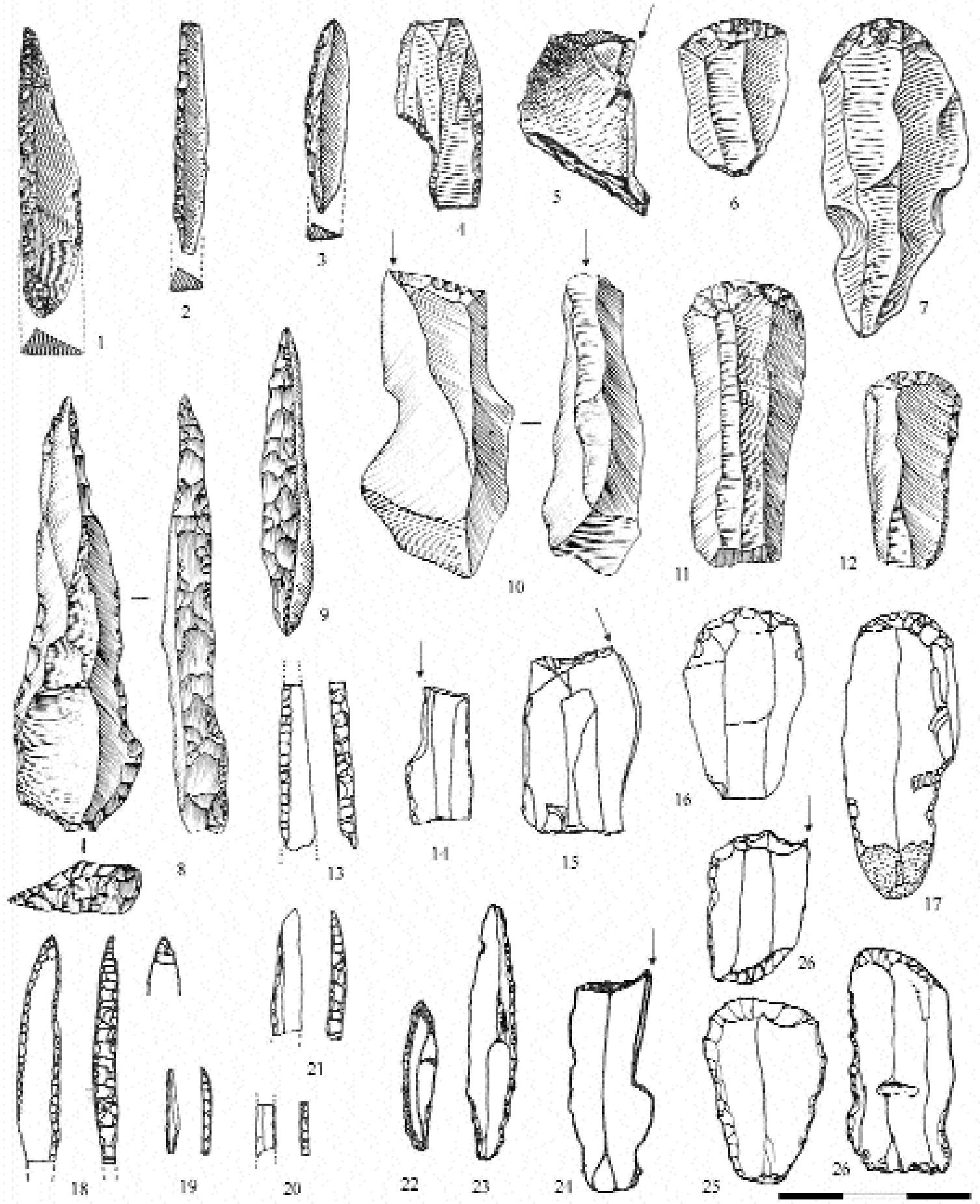

Figure 4 - Matériaux gravettiens de la grotte de Cendres. Pièces esquillées (1-3), burins (4, 7-9), grattoirs (5-6), tronqués (10), pointes “type Cendres " (11-12), outillage à dos (13-18). D'après les dessins de Villaverde et Roman (2004).

Figure 4 - Gravettian materials from Cendres cave. Flaked elements (esquillées) (1-3) ; burins (4, 7-9), end scrapers (5-6), truncations (10), points "Cendres type " (11-12), backed elements (13-18). Drawings from Villaverde and Roman (2004). 
cinq grottes : Cendres (Moraira, Alacant), le Parpalló (Gandia, València), les Malladetes (Barx, València), Beneito (Muro d'Alcoi, Alacant) et Barranc Blanc (Ròtova, València) (tabl. 1). Les autres sites ayant fourni des matériaux et des niveaux gravettiens ne seront pas traités dans ce texte pour diverses raisons qui limitent leur étude ${ }^{6}$

\section{La Cova de Cendres (Teulada-Moraira)}

Les niveaux XIV à XVI, fouillés jusqu'à maintenant dans un sondage de $2 \mathrm{~m}^{2}$ et d'une profondeur de 70 et $80 \mathrm{~cm}$, ont foumit une riche industrie du Gravettien (Villaverde y Roman 2004), soutenue par une série de quatre datations radiocarbone qui situent la base actuelle de la séquence à $25850 \pm 260$ BP. Les données paléoclimatiques et économiques sont en cours d'étude et nous limiterons nos commentaires à l'industrie lithique et osseuse.

Etant donnée la superficie fouillée réduite, le nombre de pièces est insuffisant pour établir des considérations sur l'évolution du Gravettien sur ce site ; un travail qui sera abordé quand l'extension de la fouille aura avancé. Les effectifs des niveaux XIV à XVI sont représentés par 2088 restes lithiques parmi lesquels 1162 correspondent à des fragments et 123 exemplaires sont retouchés. Malgré le faible nombre de pièces, il est possible de pressentir une évolution dans certains traits technologiques et typologiques, dont la caractérisation sera approfondie dans le futur (fig. 4).

La matière première prédominante est le silex $(98,7 \%)$. Les supports microlaminaires sont plus abondants dans les niveaux les plus profonds et leurs longueurs se situent entre 20 et $30 \mathrm{~mm}$, alors que les supports laminaires dépassent difficilement $45 \mathrm{~mm}$, situant la grande majorité entre 20 et $35 \mathrm{~mm}$. Les talons sont généralement lisses et punctiformes ou linéaires.

Parmi les 24 nucléus, 15 sont informes, ne permettant pas de précisions excessives bien que nous observions une certaine diversification des types avec un équilibre entre les unipolaires et les bipolaires. Les nucléus sont préférentiellement choisis parmi les éclats au détriment des nodules.

Avant de présenter le matériel retouché de Cendres, nous avons choisi d'exclure le niveau XIV, bien que nous croyons qu'il s'agisse d'un niveau du Gravettien final daté de $21230 \pm 180 \mathrm{BP}$, il n'est pas exclu d'envisager des contacts avec le niveau supérieur, marqué notamment par une pointe à face plane. Les fouilles en cours permettront de mieux définir ce niveau.
L'ensemble du matériel des niveaux XV et XVI (subdivisé en $A, B$ et $C$ ) offre des traits typologiques assez classiques par rapport au Gravettien d'autres régions d'Espagne et d'Europe : l'indice de burins $(7,84 \%)$ est supérieur à celui des grattoirs (2,94\%), les premiers présentent une certaine diversité typologique marquée notamment par la présence de burins plans et sur troncature; les pièces à dos sont abondantes (19,6\%) avec une concentration de gravettes, microgravettes et de "pointes type Cendres" que des pièces microlaminaires $(26,47 \%)$ comme des lamelles à dos simples et appointées.

Au sein de ce matériel, nous pouvons noter deux traits qui individualisent les industries de Cendres. Le premier correspond aux pointes "type Cendres" et le second trait particulier provient des pièces esquillées, non mentionnées jusqu'ici dans d'autres séries de la région valencienne, qui forment une part importante $(11,8 \%)$ surtout dans le niveau XVIA. Une donnée que nous retrouvons uniquement dans les niveaux gravettiens de Reclau Viver (Serinyà, Girona) (Soler 1986).

$\mathrm{Au}$ niveau technologique, il est intéressant de signaler l'existence dans les niveaux XV et XVI de l'utilisation de lames et lamelles à crête unilatérale pour la réalisation de gravettes et microgravettes. Pour cela, les supports sont légèrement retouchés, rejoignant le demi dos formé par la crête. Les retouches secondaires, parfois inverses, permettent de confirmer la volonté de ce procédé.

Un autre fait contredit les données de la séquence de Malladetes (Fortea et Jordà 1976 ; Miralles 1982b ; Fortea et al. 1983). En effet, à Cendres, l'outillage microlaminaire apparaît plus riche et diversifié dans les couches profondes, avec la présence de lamelles à dos très fines, déjà observées à la grotte Beneito (Iturbe et Cortell 1982 et 1992).

Un autre apport des fouilles de ce gisement correspond à l'industrie osseuse et la parure. La première est peu variée typologiquement, avec une nette dominance des pointes doubles longues à base arrondie ou polygonale, fines et généralement en os. L'importance du nombre de pièces par rapport à la faible superficie fouillée, tend à relativiser l'hypothèse d'une augmentation de l'industrie osseuse au Solutréen. De plus, différents types de gastéropodes perforés et des dentales (dentalium) ainsi que deux canines de cerfs à perforation médiane complètent l'ensemble.

\section{La grotte du Parpalló (Gandia)}

La série de la grotte du Parpalló est très réduite et correspond à la première occupation du site. L'industrie,

(6) Parmi ces gisements, nous pouvons citer la grotte de les Meravelles (Gandia, València) Villaverde 1979), la grotte dels Porcs (Real de Gandia, València) (Aparicio et al. 1983), la Ratlla del Bubo (Crevillent, Alacant) (Miralles 1982 ; Menargues y Navarro 2001), la grotte del Sol (Fondó de les Neus, Alacant) (Miralles 1982), la grotte de les Calaveres (Benidoleig, Alacant) (Aparicio et al. 1982), la grotte del Fontanal (Onil, Alacant) (González Prats 1982) et la grotte del Xorret (Crevillent, Alacant) (Serna 1991).

(7) Nous nous référons à des lames appointées relativement épaisses, longues et disymétriques, portant des retouches simples sommaires, souvent partielles, qui profitent de la morphologie du support pour renforcer son appointement. 

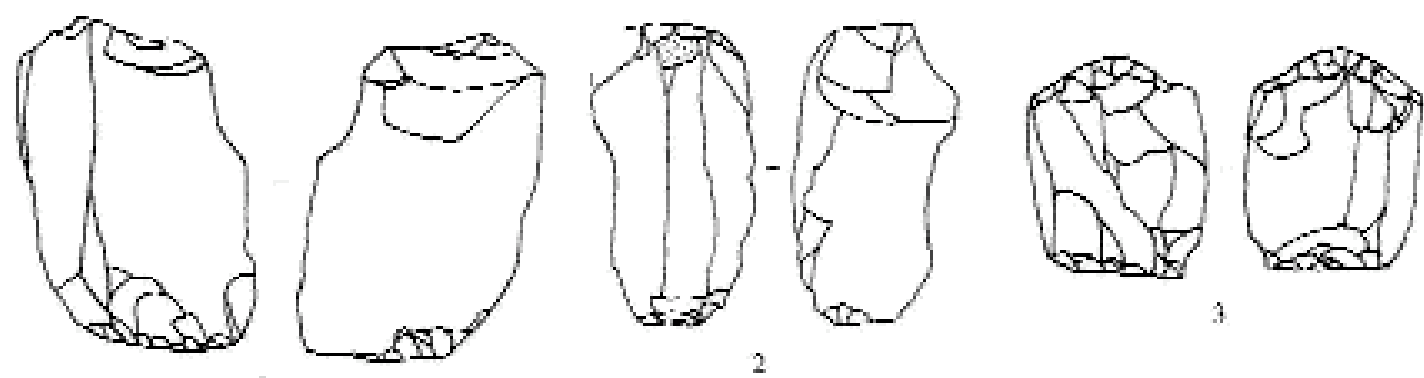

3
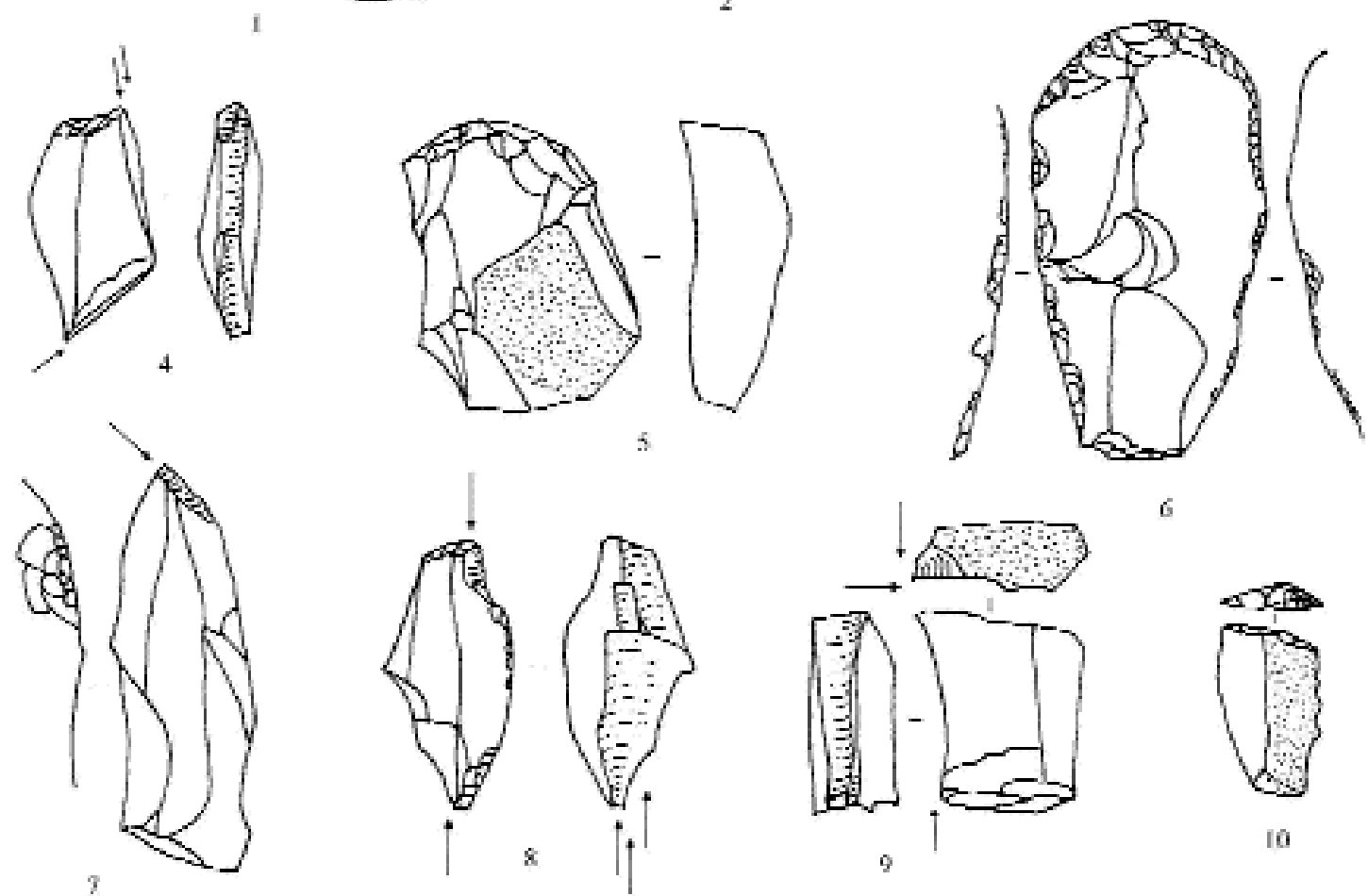

6
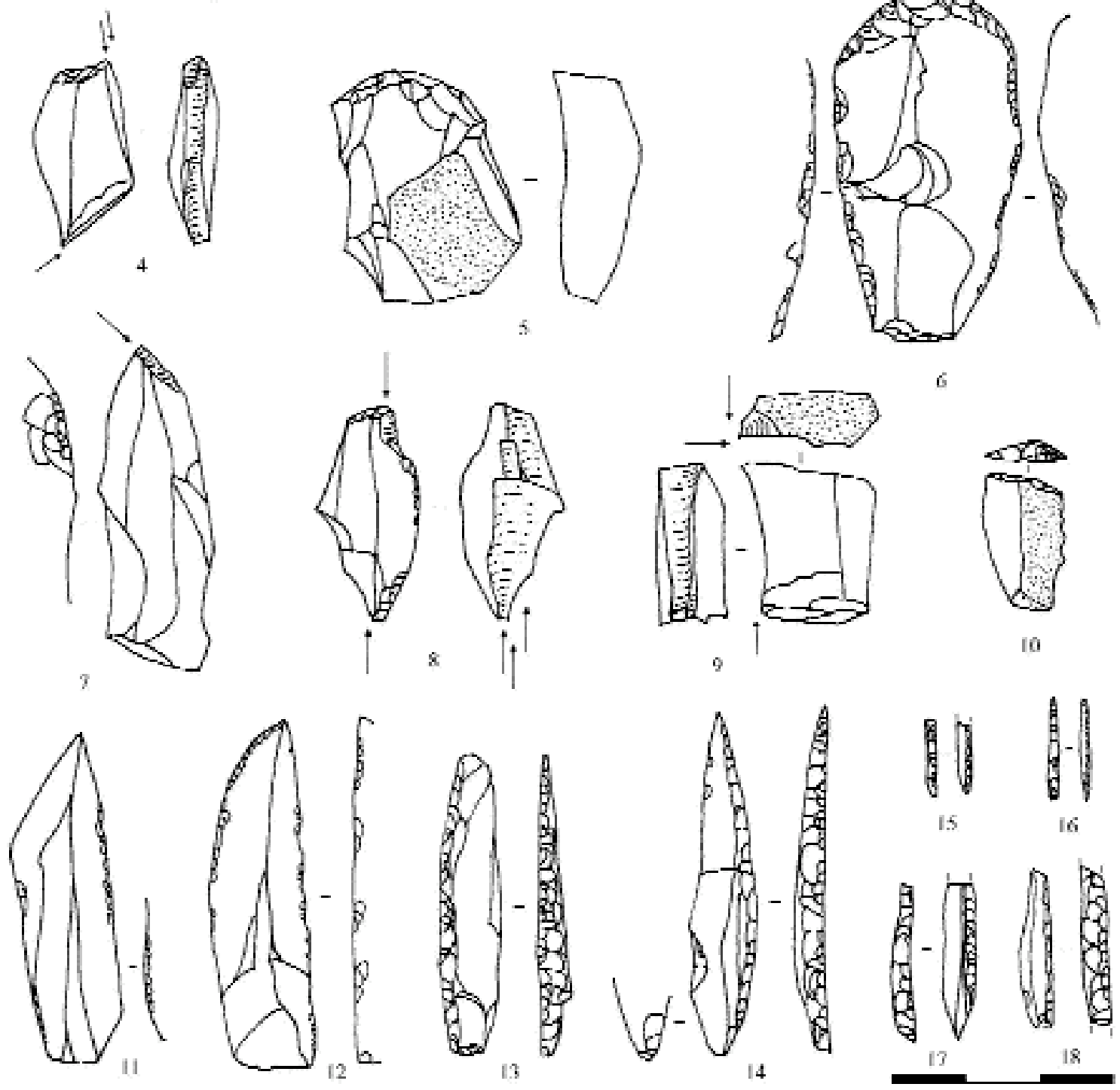

Figure 5 - Matériaux gravettiens de la grotte de Parpalló (1-7), Malladetes (8-12), Cova Beneito (13-21) et Barranc Blanc (2226). Gravettes, microgravettes et lamelles à dos (1-3, 8-9, 13, 18-23); cran (4); burins (5, 10, 14-15, 24, 26); grattoirs (6-7, 11 12, 16-17, 25-26). D’après les dessins de Pericot (1942), Fortea et Jordà (1976), Iturbe et al. (1993) et Fullola (1979).

Figure 5. Gravettian materials from Parpalló cave (1-7), Malladetes cave (8-12), Cova Beneito (13-21) and Barranc Blanc (2226). Gravettes, microgravettes et backed bladelets (1-3, 8-9, 13, 18-23); shouldered points (4); burins (5, 10, 14-15, 24, 26); end scraspers (6-7, 11-12, 16-17, 25-26). From drawings ofPericot (1942), Fortea and Jordà (1976), Iturbe et al. (1993) and Fullola (1979). 
connue par les travaux de Fullola (1978) et Miralles (1982), se réduit à 86 pièces retouchées. II s'agit d'une industrie lamino-lamellaire, où contrairement à Cendres, l'indice des grattoirs est élevé $(55,81 \%)$ par rapport aux burins $(3,48 \%)$, tous dièdres. Les pièces à dos, dont des gravettes, microgravettes, lames et lamelles à dos tiennent une place importante $(25,58 \%)$. Aucune pièce esquillée n'est mentionnée (fig. 5). Parmi l'industrie osseuse, il faut noter l'existence de pointes à base volumineuse et de certaines pointes de section circulaire.

Tout ce matériel provient, selon Pericot (1942), de deux moments de forte concentration de restes situés autour de 8 et $7,25-7,75 \mathrm{~m}$ de profondeur.

\section{La grotte de Les Malladetes (Barx)}

Le matériel gravettien de la grotte de Les Malladetes, qui provient de fouilles des années 1940, a permis de différencier, à partir de légères variations des indices microlaminaires et de pièces à dos, deux phases d'évolution (Fortea y Jordà 1976 ; Miralles 1982b ; Fortea et al. 1983).

Comme à Cendres et contrairement au Parpalló, Malladetes présente de riches niveaux gravettiens. Selon Miralles (1982a), le nombre de pièces retouchées (couches 46 à 49) est de 486 parmi lesquelles on trouve des gravettes, microgravettes, lames et lamelles à bord abattu en pourcentages importants (fig. 5).

En divisant la puissance du remplissage en deux unités, nous pouvons observer que les couches inférieures (de 2,4 à $3,2 \mathrm{~m}$ ) présentent un indice de pièces à dos de $14,17 \%$ alors que dans les niveaux supérieurs (de 1,6 a 2,4 m), il est de 23,03\%. Les deux niveaux sont dominés par les grattoirs $(35,07 \%$ et $35,94 \%)$ et les pièces à retouches continues $(24,62 \%$ et $22,12 \%)$, quelques burins $(7,08 \%$ et $5,99 \%)$ et des pièces tronquées $(2,61 \%$ et $0,46 \%)$. Les pièces esquillées sont présentes dans des pourcentages inférieurs à ceux de Cendres $(2,61 \%$ et 2,30\%).

Les gravettes et micro-gravettes suivent le chemin inverse et bien que les premières soient nombreuses dans les couches inférieures, les secondes dominent dans les couches supérieures. Cette donnée, comme ce que nous avons dit auparavant, est contraire à ce que l'on observe à Cendres ou à Beneito, où l'outillage microlaminaire est plus abondant dans les niveaux inférieurs de la séquence (Nillaverdey Roman 2004 ; Iturbe y Cortell 1982 y 1994).

L'unité inférieure a été datée (Secteur E, couche 12 (2,75$2,9 \mathrm{~m})$ ) à $25120 \pm 240 \mathrm{BP}$ (Villaverde 2001) ; une date proche du sous-niveau XVIC de la grotte de Cendres.

Les résultats obtenus par Fortea et Jordá (1976), pendant la campagne réalisée en 1971, confirment l'entité gravettienne et les traits basiques de l'évolution évoquée précédemment. Cela correspond au Gravettien des niveaux VIII-X de la Cata Este, au- dessus d'un niveau du Solutréen initial.

\section{La grotte de Beneito (Agres)}

Les matériaux gravettiens de la grotte Beneito présentent une meilleure précision stratigraphique que les deux précédents (Iturbe et al. 1994) et des informations abondantes concernant cette phase industrielle (niveaux B7a, B7b et, sans doute, B6). L'industrie est assez uniforme mais présente quelques variations : les grattoirs représentent un pourcentage supérieur aux burins, les pièces à retouches continues, à la différence du reste des gisements, comme les pièces esquillées sont peu nombreuses (fig. 5).

Si l'on observe plus précisément les pièces à dos, nous pouvons voir une évolution considérable dans la valeur des pièces laminaires et lamellaires. Comme à Cendres, l'outillage microlaminaire est plus abondant et varié dans les niveaux inférieurs, avec la présence de lamelles à dos, très fines, normées, dans le niveau B7b et des lamelles à retouches inverses, signalées par ailleurs dans l'étude du gisement réalisée par Iturbe et Cortell (1982 et 1984).

\section{La grotte de Barranc Blanc (Ròtova)}

Fouillée dans les années 1950 et 1970, les matériaux de ce gisement posent certains problèmes stratigraphiques et des mélanges avec le matériel solutréen de certaines couches (principalement la 6) sont possibles (Fletcher 1953 ; Fullola 1978 ; Miralles 1982).

Sans oublier ces circonstances et à partir des données de Miralles (1982), il est possible d'observer que parmi les pointes à dos des couches 7 à 9 , ne sont présentes que des microgravettes $(27,8 \%)$; les pièces à retouches continues $(27,8 \%)$ sont abondantes, les burins tiennent une place modérée $(12,83 \%)$ et en général, les pièces à dos, laminaires ou microlaminaires $(5,88 \%$ et $2,13 \%)$ sont peu représentées (fig. 5). La comparaison de ces données avec celles des niveaux XIV de Cendres et du niveau 6 de Beneito pourrait suggérer une chronologie avancée pour le Gravettien de ce site.

\section{LE GRAVETTIEN DE LA RÉGION DE MURCIA ET EN ANDALOUSIE}

Dans la partie sud de la façade méditerranéenne, les données sont plus limitées. Le Paléolithique supérieur initial dans ces zones littorales a été révisé par Cacho (1980 et 1982), qui s'est appuyée principalement sur les collections de L. Siret. Aux gisements cités dans cette oeuvre, parfois avec réserves (Fortea 1986), peut être ajoutée la grotte de Nerja (Nerja, Málaga, Jordá 1986 ; Aura et al. 1998), et l'ensemble de Bajondillo (Torremolinos, Málaga, Cortés et Simón 1995, 1998 ; Cortés 2002).

Nous pourrions retenir également comme Gravettien, les matériaux de Zájara, à partir de la présence de quelques pièces à dos ; ceux de Morote et les niveaux inférieurs de Serrón. En général, les collections de Murcia et d'Almería se caractérisent par la faiblesse numérique du matériel à partir duquel il n'est pas possible d'approfondir la caractérisation. 
La collection de Bajondillo est très réduite et correspond au niveau 10. Les pièces retouchées sont peu nombreuses, dominées par les burins, plus abondants que les grattoirs, des éléments à dos comme des gravettes et micro-gravettes et quelques pièces tronquées. Nous tenons à souligner la présence occasionnelle d'outils micro-laminaires.

La grotte de Nerja offre, dans la Sala del Vestíbulo (niveaux 11 à 13), un ensemble de pièces caractérisées par la taille laminaire, avec des supports de bonne dimension sur lesquels sont réalisés des grattoirs sur bout de lame, des burins et des lamelles à dos parfois bipolaires, des pièces tronquées et une pointe à retouche simple mais également quelques pointes en os et de la parure.

\section{SYNTHÈSE RÉGIONALE}

En général, les données disponibles pour le Gravettien méditemanéen sont réduites et les contextes stratigraphiques souvent mal définis. Ces limites, qui sont parfois le produit de l'ancienneté des fouilles ou de la faiblesse numérique des ensembles industriels, sont déterminantes au moment d'établir des conclusions. Les niveaux XV et $\mathrm{XVI}$ de Cendres proviennent d'un sondage pour lequel il faut donc rester prudent quant aux interprétations. Les ensembles d'une certaine importance sont le Reclau Viver et l'Arbreda en Catalogne, Parpalló, Malladetes, Beneito et Cendres en Pays valencien.

L'origine du Gravettien méditerranéen ibérique constitue une question qui reste ouverte aujourd'hui. Les datations absolues de la zone septentrionale, peu nombreuses, se situent dans des contextes avancés et seules les zones centrales et méridionales présentent des dates anciennes. Par ailleurs, le nombre de sites avec des niveaux aurignaciens a augmenté ces demières décennies mais le panorama est très orienté vers la zone septentrionale où les riches séquences de l'Arbreda et les données de Reclau Viver permettent de confirmer l'importance de cette phase dans la séquence du Paléolithique supérieur régional. Pour autant, entre les dates de l'Aurignacien initial et celles du début du Gravettien, se déroulent des millénaires. II est seulement possible de se référer à des ensembles réduits, souvent pauvres en matériel ou de chronologie imprécise. L'antiquité des dates obtenues dans certains contextes méridionaux paraît avaliser l'hypothèse d'une extension rapide de l'Aurignacien pour tout le versant méditerranéen ibérique. Cependant, les séquences sont rares pour observer, à partir de l'Aurignacien initial ou ancien, des stratigraphies ou des étapes de son évolution. II est possible que cette situation soit en partie le résultat d'un manque de prospections ou d'une caractérisation inadéquate de certains ensembles ramassés dans des sites de plein air ; cependant, il s'agit d'un panorama différent de ce qui se produit avec le Gravettien. La zone catalane se distingue du fait que la sériation de l'Aurignacien offre une meilleure entité et continuité (Fullola et al. sous presse).

Les séquences stratigraphiques présentant la succession Aurignacien et Gravettien sont peu nombreuses dans le
Pays valencien et en Andalousie mais permettent de caractériser les industries qui ne présentent pas de discontinuité chronologique. Les exemples de Malladetes, Bajondillo et Ratlla del Bubo sont significatifs : les collections sont réduites ou la provenance des matériaux insatisfaisante. La date de l'Aurignacien de Malladetes, 29690 \pm 560 BP, s'éloigne du Gravettien daté de $25120 \pm 240$ BP. Seul le site de Beneito offre une bonne succession entre l'Aurignacien évolué et le Gravettien, mais nous manquons de datations absolues pour cette série. Les mêmes occurrences sont visibles à la Ratlla del Bubo où le Gravettien est connu uniquement grâce à des collections privées. Tout cela réduit beaucoup les possibilités d'établir un rapprochement entre les deux phases. En Catalogne, les séquences de l'Arbreda et Reclau Viver permettent de mettre en valeur cette question.

En tenant compte de son emplacement sur les mêmes sites de Serinyà, et l'utilisation des mêmes matières premières que les aurignaciens, on a l'impression dans cette région, que le Gravettien constitue une évolution naturelle de l'Aurignacien. Pour voir une solution de discontinuité dans la séquence du Paléolithique supérieur en Catalogne, il faudra attendre le Magdalénien avec l'abandon des gisements du Reclau de Serinyà, l'utilisation massive du silex pour toute l'industrie lithique et la grande importance accordée à l'industrie osseuse. On peut supposer que sur un substrat aurignacien évolué polymorphe et tardif, avec généralement beaucoup des burins de différents types, sans doute appuyé par des influences extérieures, a été formé le Gravettien qui peut contenir dans ces moments initiaux, un nombre important de burins. Si le Gravettien apparaît après l'Aurignacien tardif avec beaucoup de burins, on peut penser à un début du Gravettien où les burins ont une grande importance et sont plus abondants que les grattoirs, et, on peut alors, regrouper les gisements gravettiens sous cette caractéristique, comme par exemple Castell sa Sala.

Par ailleurs, les traits permettant de définir les caractéristiques du Gravettien méditerranéen ibérique s'appuient sur le fait que les industries gravettiennes présentent une considérable uniformité dans tout l'ensemble méditerranéen péninsulaire, surtout dans les moments initiaux du Gravettien méditerranéen, entre 26000 et 21000 BP. Cette région reste différente de ce qui est actuellement établi dans les complexes gravettiens du nord de la péninsule, du sud-est de la France ou d'une partie de I'Italie. Avec le Gravettien, est documenté l'apparition de l'art dans les séquences du Parpalló et de Malladetes ; il existe également des indices de quelques figures d'art pariétal andalou qui pourraient remonter à cette étape.

L'uniformité qu'offre le Gravettien sur tout le versant méditerranéen, conséquence de l'inexistence de grandes transformations dans sa structure industrielle tout au long de la séquence, est cohérente avec l'idée que son origine réponde à une stimulation ponctuelle, préalable à l'expansion des industries gravettiennes à burins de Noailles, et que son expansion sur le versant méditerranéen péninsulaire a du se produire, de manière assez rapide, vers 27 000-26 000 BP. 


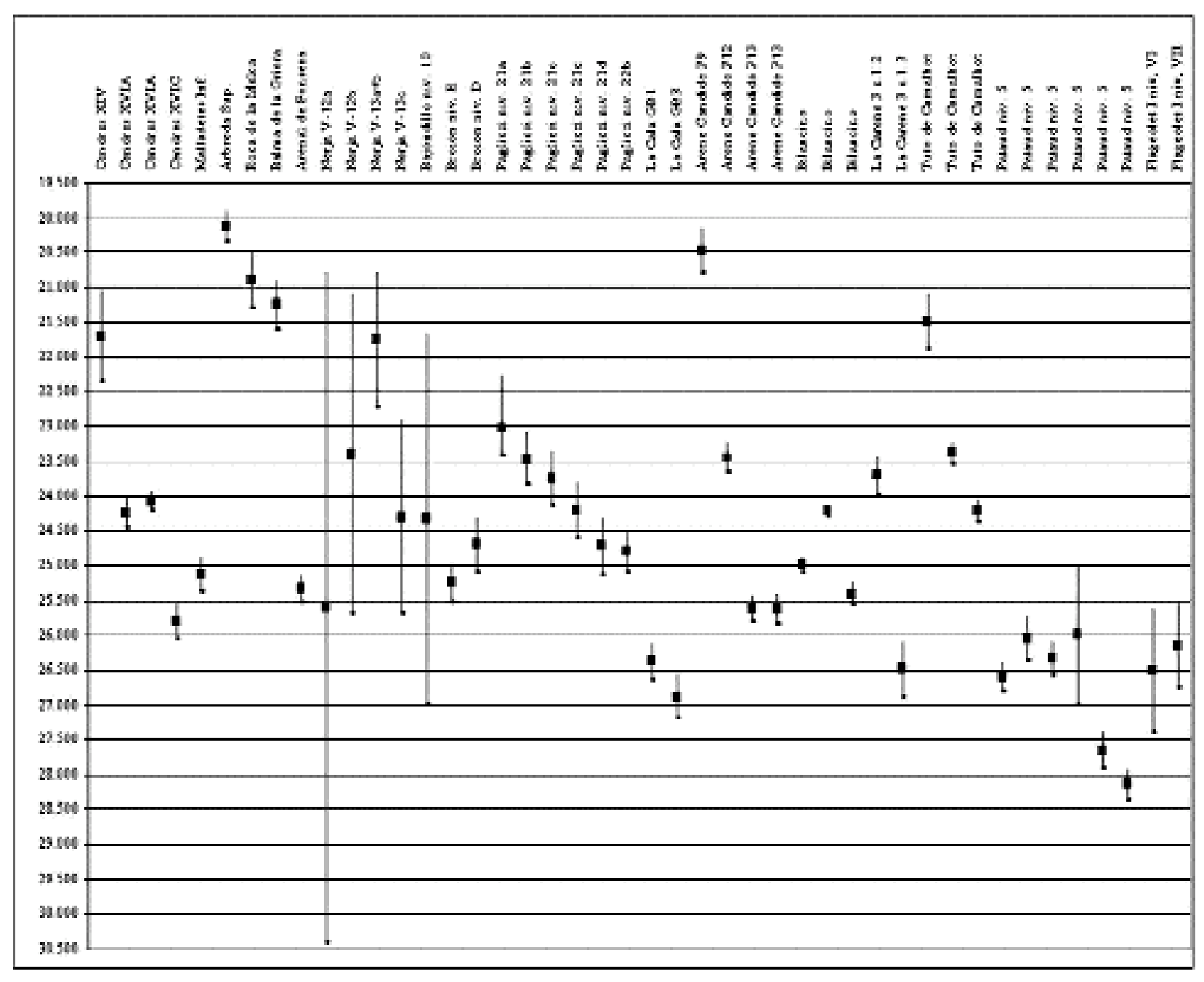

Figure 6 - Datations du Gravettien méditerranéen ibérique.

Figure 6 - Dates of iberian mediterranean Gravettian.

Comme nous l'avons déjà indiqué, mesurer le poids du substrat culturel autochtone, sur lequel se développe apparemment de manière rapide et homogène le Gravettien dans l'ensemble méditerranéen, présente une grande difficulté d'analyse. II ne semble pas que les variations des indices de burins ou de l'outillage microlaminaire soient significatifs dans l'ambiance valencienne. Une explication séquentielle ne doit pas être considérée comme le résultat de la coexistence de traditions industrielles gravettiennes distinctes dans cette région. C'est pour cela que nous considérons que le polymorphisme typologique du Gravettien de cette zone peut s'expliquer en termes fonctionnels, comme le suggère la fluctuation observée des pièces esquillées.
Dans cette explication interviennent, sans doute, des facteurs de spécialisation de la chasse et d'une haute mobilité territoriale, illustrée par la faune des niveaux gravettiens en Pays valencien, qui favorisent les ensembles industriels reflétant des activités de caractère stationnaire. De plus, la forte mobilité territoriale est cohérente avec l'existence d'un niveau démographique bas, propice d'autre part à l'existence de nombreux réseaux sociaux ouverts. II s'agit d'une des conditions idoines pour modéliser l'expansion du Gravettien ou pour rendre compte des coïncidences stylistiques dans les systèmes de parure personnelle, comme on le voit dans les sépultures ou dans le style des représentations artistiques figuratives pariétales et mobilières de ces dates en péninsule ibérique (Zilhão 2003). 
Les niveaux $X V$ et $X V I$ de Cendres contribuent également à clarifier l'évolution du Gravettien de la zone centrale méditemanéenne. Jordá (1954: 17), à partir des résultats de fouilles de Malladetes et du Parpalló, a signalé une tendance vers le microlithisme dans les niveaux supérieurs du Gravettien valencien, avec une meilleure présence des lamelles à dos, et surtout de microgravettes. Cette même tendance, sans préciser l'importance des lamelles à dos, sinon comprises comme microgravettes, fut réitérée des années après par Jordá et Fortea (1976: 140) à partir de résultats obtenus dans la fouille de l'année 1970 à Malladetes. Pour autant, les données offertes par Beneito et Cendres contredisent cette idée concernant l'outillage microlaminaire. Avant la fouille de Cendres et de Beneito, le Gravettien régional était défini par un indice faible de burins par rapport aux grattoirs et un outillage dominé par les dos de petite taille (microgravettes, lamelles à dos simples et appointées), avec une faible part de pièces tronquées, à peine nuancée dans les moments plus avancés. Cendres présente un meilleur indice de burins de différents types, un faible indice de grattoirs et un pourcentage élevé de pièces esquillées. Les indices de pièces à dos n'offrent pas de variations comparables au Parpalló ou à Malladetes, comme l'importance relative de pièces à retouches continues sur les deux bords. Les troncatures sont peu abondantes même dans les moments plus récents. L'outillage microlaminaire de Cendres et de Beneito est plus important dans la partie inférieure de la séquence ( q.1). Certaines circonstances atténuent les différences avec les ensembles gravettiens du Nord et Sud de la péninsule et contribuent à augmenter la sensation que nous sommes avant l'étape d'uniformisation sur tout le versant méditerranéen ibérique.

L'industrie osseuse de Cendres présente une certaine singularité par la typologie de ses pièces où dominent les pointes doubles longues sur supports osseux, sinon par l'unité atteinte à certains moments. II n'existe pas d'autres références de cette unité dans d'autres gisements, mais Cendres confirme qu'il ne faut pas attendre le Solutréen pour considérer que l'os constitue une matière première importante.

En ce qui concerne la chronologie, les datations disponibles pour le Gravettien du versant méditerranéen sont récentes pour la zone catalane, proches des étapes finales du Gravettien régional, et ambiguës pour la zone andalouse, comme celles obtenues à Nerja. Dans ce gisement, la publication d'une nouvelle date (Arribas et al. 2004), de 24 $480 \pm 110 \mathrm{BP}$, confirme l'antiquité du début de la séquence gravettienne de la zone méridionale qui se situe dans un espace chronologique très proche de celui qui correspond au sous-niveau XVIA de Cendres.

La date du sous-niveau XVIC de Cendres contribue également à préciser l'ancienneté du Gravettien dans la zone centrale de la région méditerranéenne péninsulaire au moins jusqu'à environ 26000 BP. Cette datation, associée aux deux autres obtenues dans le sous-niveau XVIA de Cendres, couche 12 du secteur E de Malladetes (campagne de 1948) et celle obtenue dans les niveaux d'Arenal de Fonseca (Utrilla et Domingo 2001-2002), permettent de préciser les dates des phases les plus anciennes du Gravettien régional (fig.6). L'ensemble de dates de Cendres, Malladetes y d'Arenal de Fonseca, renvoient à l'intervalle de 24000 - 26000 BP pour une étape caractérisée par l'abondance des pièces à dos et une composition industrielle cohérente par rapport au Gravettien indifférencié français (Bosselin et Djindjian, 1994) ou au Gravettien ancien (Djindjian 2000), dont les datations peuvent se situer à partir des dates obtenues à Pataud et au Flageolet I entre 26000 et 27000 BP. Ces résultats sont cohérents avec l'expansion de ces faciès industriels dans des dates antérieures aux industries à Noailles (Otte 2003). Cela est suggéré par les datations des niveaux inférieurs du Gravettien de Paglicci (Palma di Cesnola 2001), de La Cala (Boscato et al. 1997), de Broion (Broglio et Impronta 199495), ou celles du Gravettien des Pyrénées centrales françaises (Foucher et al. 2001). Finalement, cela reste pour le moment situé entre l'horizon des dates de l'Arbreda, du Roc de la Melca et de la Griera, proches du niveau XIV de Cendres, et du Solutréen inférieur de Malladetes ou du Parpalló, c'est-à-dire, dans une limite temporelle qui ne semble pas dépasser 21000 - 20500 BP.

Etablir le degré de relation entre ce Gravettien final méditerranéen ibérique et le Solutréen demeure problématique, surtout pour les ensembles solutréens inférieurs. Intervient probablement l'appauvrissement que représente l'occupation finale du Gravettien de Malladetes (Fortea y Jordá 1976), visible dans le niveau VII de Cata Este ou la présence de ruptures stratigraphiques comme à Cendres, Nerja ou Bajondillo. Une circonstance qui peut, selon nous, s'étendre à Beneito et au Reclau Viver, et pourrait expliquer la rareté des datations gravettiennes entre $21000-24000$ BP.

A Cendres, indépendamment de l'exacte attribution industrielle du niveau XIV, il existe une rupture sédimentaire associée à une industrie du Solutréen évolué entre ce niveau et le XIII. Nerja offre, dans la séquence de la Sala del Vestíbulo, une situation très proche. Entre les niveaux 11 à 13, en relation avec le Gravettien et l'ensemble des niveaux 8 à 10 attribués au Solutréen moyen et supérieur, est enregistré un processus érosif (Aura et al. 1998). La séquence de Bajondillo se caractérise aussi par un passage, du Gravettien (niveau 10) à un Solutréen moyen (niveau 9), marqué par des problèmes d'attributions industrielles stratigraphiques (Cortés 2002). En ce qui concerne Beneito (Iturbe et al. 1994), nous sommes enclins à interpréter l'attribution du niveau 6 au Gravettien final, expliquant la présence de quelques pièces à retouches solutréennes comme le résultat de mélanges avec l'unité supérieure, en relation avec le Solutréen évolué (Villaverde et al. 1998). Une lecture assez semblable pourrait faire de l'industrie assignée par Soler (1994) au Proto-solutréen du Reclau Viver, le niveau E, qui se caractérise par la présence de pièces bifaciales. Finalement, le premier niveau solutréen de l'Arbreda offre des problèmes de définition similaires, sachant que parmi les pièces solutréennes, on observe la présence d'une pointe à cran à retouches solutréennes (Soler et Maroto 1987 : 223) qui invalide la possibilité d'une antériorité du niveau $\mathrm{E}$ aux étapes initiales du Solutréen. 
Le panorama que nous achevons de décrire coïncide pour autant avec l'idée que les phénomènes érosifs permettent d'expliquer, au moins en partie, les difficultés pour localiser les industries des premières étapes du Solutréen et aussi du Gravettien final.

\section{BIBLIOGRAPHIE}

ALCALDE G. 1987 - Els rosegadors del Paleolític Superior de la cova de l'Arbreda (Serinyà, Catalunya). Significació paleoecològica i paleoclimàtica, Cypsela, VI, Girona, pp. 89-96.

APARICIO J., GURREA V. et CLIMENT S. 1983 - Carta Arqueológica de la Safor. Instituto de Estudios Comarcales Duque Alonso el Viejo. Gandia.

APARICIO J., PÉREZ RIPOLL M., VIVES E., FUMANAL M.P. et DUPRÉ M. 1982 - La Cova de les Calaveres (Benidoleig, Alicante). Trabajos Varios del S.I.P., 75.

ARRIBAS A., AURA J.E., CARRIÓN J.S., JORDÁ J.F. et PÉREZ RIPOLL M. (2004): "Presencia de hiena manchada en los depósitos basales (Pleistoceno superior final) del yacimiento arqueológico de la Cueva de Nerja (Málaga, España)". II Congreso Ibérico de Paleontología / XVIII Jornadas de la Sociedad Española de Paleontología.

AURA TORTOSA J.E., JORDÁ PARDO J.F., GONZÁLEZTABLAS J., BÉCARES PÉREZ J. et SANCHIDRIÁN TORTÍ J.L. 1998 - Secuencia arqueológica de la Cueva de Nerja: la Sala del Vestíbulo. Las culturas del Pleistoceno Superior en Andalucía, pp. 217-236.

BISCHOFF J.-L., K. LUDWIG, J. F. GARCÍA, E. CARBONELL, M. VAQUERO, T. W. STAFFORD et J. T. JULL (1994): Dating of the Basal Aurignacian Sandwich at Abric Romaní (Catalunya, Spain) by Radiocarbon and Uranium-Series, Journal of Archaeological Science, 21, pp. 541-551.

BOSCATO P., RONCHITELLI A. et WIERER U. 1997 - "॥ Gravettiano antico della Grotta della Cala a Marina Camerota". Rivista di Scienze Preistoriche, 48, pp. 97-186.

BOSSELIN B. et DJINDJIAN F. 1994 - "La Chronologie du Gravettien Français”. Préhistoire Européenne, 6, pp. 77-115.

BROGLIO A. et IMPRONTA S. 1994-1995 - "Nuovi dati di cronologia asoluta del Paleolitico superiore e del Mesolitico del Veneto, del Trentino e del Friuli".

BURJACHS F. et RENAULT-MISKOVSKY J. 1992 Paléoenvironnement et paléoclimatologie de la Catalogne durant près de 30,000 ans (du Würmien ancien au début de l'Holocène) d'après la Palynologie du site de l'Arbreda (Gérone, Catalogne), Quaternaire, 3, 2, pp. 75-85.
BURJACHS F. 1993 - Paleopalinología del paleolítico superior de la Cova de l'Arbreda (Serinyà, Catalunya), Estudios sobre el Cuaternario. Medios sedimentarios. Cambios ambientales. Hábitat humano. Fumanal, M.P. \& Bernabeu, J. (eds.), Valencia, pp. 149-157.

CACHO C. 1980 - Secuencia cultural del Paleolítico superior en el sureste español. Trabajos de Prehistoria, 37, pp. 65-108. CACHO C. 1982 - El paleolítico superior del Levante español en su contexto del Mediterraneo occidental (S.E. de Francia e Italia). Itálica, 16, pp.7-32.

CEBRIA A., ESTEVE X.et VILARDELL A. 2000 - Cavalls, cérvols i conills. 10 anys de recerca a la balma de la Graiera de Calafell. El congost de la Cobertera a la Prehistòria, ed. Mairie de Calafell, 27 pp., 4 fig., Calafell.

COROMINAS J.M. 1946 - La cueva del Reclau Viver de Seriñá, Anales del Instituto de Estudios Gerundenses, I, Gerona, pp. 209-223.

COROMINAS J.M. 1949 - El Paleolítico superior en la cueva «Reclau-Viver» de Seriñá (España), Rivista di Scienze Preistoriche,IV, 1-2, Firenze, 1959, pp. 43-54.

CORTÉS M. 2002 - El Paleolítico medio y superior en el sector central de Andalucía (Córdoba y Málaga). Tesis doctoral. Universidad de Córdoba.

CORTÉS M. et SIMÓN M. 1995 - Cueva Bajondillo (Torremolinos, Málaga). Aportaciones al Paleolítico en Andalucía. El Món Mediterrani després del Pleniglacial (eds J.M. Fullola \& N. Soler). Serie Monográfica 17, Girona.

CORTÉS M. et SIMÓN M.D. 1998 - Cueva Bajondillo (Torremolinos, Málaga), implicaciones para el conocimiento de la dinámica cultural del Pleistoceno superior en Andalucía. Las culturas del Pleistoceno Superior en Andalucía. Patronato de la Cueva de Nerja, pp. 35-62.

DELIBRIAS G., ROMAIN O. et LE HASIF G. 1987 Datation par la méthode du carbone 14 du remplissage de la grotte de l'Arbreda, Cypsela, VI, Girona, pp. 133-135.

DJINDJIAN F. 2000 - "The Mid Upper Palaeolithic (30,000 to $20,000 \mathrm{bp}$ ) in France". Hunters of the Golden Age. The Mid Upper Palaeolithic of Eurasia 30,000-20,000 bp. (edited by W. Roebroeks, M. Mussi \& K. Fennema). University of Leiden, pp. 313-324.

FLETCHER D. 1953 - Covacha de Barranc Blanc. Rotova (Valencia) Noticiario Arqueológico Hispano, 1, pp. 10-12.

FORTEA J. 1973 - Los complejos microlaminares y geométricos del Epipaleolítico mediterráneo español. Memorias del Sem. de Prehist. y Arqueología, 4, éd. Univ. de Salamanque, Salamanque.

FORTEA F.J. 1986 - El Paleolítico superior y Epipaleolítico en Andalucía. Estado de la cuestión cincuenta años después. Actas del Congreso Homenaje a L. Siret, pp. 67-78. 
FORTEA F.J. et JORDÁ F. 1976 - La Cueva de Les Mallaetes y los problemas del Paleolítico Superior del Mediterráneo Español. Zephyrus, XXVI-XXVII, pp. 129-166.

FORTEA F.J., FULLOLA J.M., VILLAVERDE V., DAVIDSON I., DUPRÉ M. et FUMANAL M.P. 1983 Schéma paléoclimatique, faunique et chronostratigraphique des industries à bord abattu de la région méditerranéenne espagnole. Rivista di Scienze Preistoriche, XXXVIII, pp. 21-67.

FOUCHER P., SAN JUAN C., VALLADAS H., CLOTTES J., BÉGOUËN et GIRAUD J.P. 2001 - "De nouvelles dates 14 C pour le Gravettien des Pyrénées centrales". Bulletin de la Société Préhistorique Ariège-Pyrénées, LVI, pp. 35-44.

FULLOLA J.M. 1978 - Les anàlisis tipològiques del Barranc Blanc. Archivio di Tipologia Analitica, 6, pp. 71-149.

FULLOLA J.M., BARTROLÍ R., CEBRIÀ A., BERGADÀ M.M., FARELL D. et NADAL J. 1994 - El paleolítico superior de Catalunya: El gravetiense de la balma de la Griera (Calafell, Baix Penedès, Tarragona), Actas dos Trabalhos De Antropologia e Etnologia, XXXIV, 12, Porto, pp. 35-51.

FULLOLA J.M., VILLAVERDE V., SANCHIDRIÁN J.L., AURA J.E., FORTEA J. et SOLER N. 2006 : EI Paleolítico superior mediterráneo ibérico. IV Simposio de Prehistoria Cueva de Nerja. La Cuenca Mediteránea durante el Paleolítico Superior (38.000-10.000 años), SANCHIDRIÁN J.L., MARQUEZ A.-M. et FILLOLA J.-M. (eds), pp. 192-212.

GARCIA L. 1997 - Les restes d'oiseaux des sites de Serinyà (Pays Catalans), El món mediterrani després del pleniglacial (18.000-12-000 BP). J. M. Fullola \& N. Soler eds., U.I.S.P.P., Comission VIII, Sèrie Monogràfica, 17, Museu d'Arqueologia de Catalunya. Girana, pp. 329-344.

GONZÁLEZ PRATS A 1982 - El yacimiento epigravetiense del Fontanal de Onil (Alicante), Helike, 1, pp. 69-86.

ITURBE, G. e t CORTELL, E. 1982 - Cova Beneito: Avance preliminar. P.L.A.V.-Saguntum, 17, pp. 9-44.

ITURBE G. et CORTELL E. 1992 - El Auriñaciense evolucionado en el País Valenciano: Cova Beneito y Ratlla del Bubo. Aragón/Litoral Mediterréneo. Intercambios culturales durante la Prehistoria, pp. 129-138.

ITURBE G., FUMANAL M.P., CARRIÓN J.S., CORTELL E., MARTÍNEZ R., GUILLEM. P.M., GARRALDA, M.D. et VANDERMEERSCH B. 1994 -Cova Beneito (Muro, Alicante): una perspectiva interdisciplinar. Recerques del Museu d'Alcoi, 2, pp. 23-88.

JORDÁ F.1954 - Gravetiense y Epigravetiense en la España Mediterránea.Cesaraugusta, IV, pp.7-36.

JORDÁ F. 1986 - La ocupación más antigua de la Cueva de Nerja. En La Prehistoria de la Cueva de Nerja (Málaga), pp. 197-204.

LAPLACE G. 1962 - Le Paléolithique Supérieur de l'Abri Romani, L'Anthropologie, 66, 1-2, Paris, pp. 36-43.

MENARGUES J. et NAVARRO C. 2001 - Los materiales paleolíticos de la Ratlla del Bubo (Crevillent, Alacant) en el Museo Arqueológico Municipal de Novelda. Recerques del Museu d'Alcoi, 10, pp. 17-24.

MIRALLES J.L 1982 - El Gravetiense en el País Valenciano. Tesis de licenciatura. Universitat de València.

MIRALLES J.L. 1982 - El Gravetiense en el País Valenciano. Saguntum- PLAV, 17, pp. 45-63.

MUÑOZ M. et M. CASADEVALL 1997 - Fish remains from Arbreda Cave (Serinyà, Girona), norteast Spain, and their palaeoecological significance, Joumal of Quatemary Science, 12, pp. 111-115.

OTTE M. 2003 - "L'Europe Gravettienne”. Préhistoire de l'Europe. Des origines à l'Âge du Bronze. Editions du Comité des travaux historiques et scientifiques. Paris, pp. 227-239.

PALMA DI CESNOLA A. 2001 - Le Paléolithique supérieur en Italie. Collection l'Homme des origines. Jérôme Millon.

PERICOT L. 1942 - La Cueva del Parpalló (Gandía). Consejo Suprerior de Investigaciones Científicas. Instituto Diego Velázquez, Madrid.

PERICOT L. 1963 - L'Aurignacien et le Périgordien en Espagne. Aurignac et l'Aurignacien, Centenaire des fouilles Lartet, Bulletin de la Société Méridionale de Spéléologie et Préhistoire, t. IV-IX, 1956-1959, pp. 85-92.

PEYRONY D. 1933 - Les industries aurignaciennes dans le bassin de la Vézère. Bulletin de la Société Préhistorique Française, 30, fasc. 10, Paris, pp. 543-559.

PEYRONY D. 1936 - Le Périgordien et l'Aurignacien (nouvelles observations), Bulletin de la Société Préhistorique Française, 33, Paris, pp. 616-619.

PEYRONY D. 1946 - Une mise au point au sujet de l'Aurignacien et du Périgordien, Bulletin de la Société Préhistorique Française, 43, Paris, pp. 232-237.

ROS M.T. 1987 - Anàlisi antracològica de la cova de l’Arbreda, Cypsela, VI, Girona, pp. 67-71.

SACCHI D., SOLER N., MAROTO J. et DOMÈNECH E. 1996 - La question de l'Aurignacien tardif dans le domaine méditerranéen nord-occidental, L'Aurignacien tardif, colloque XI, XIII Congrès de I'U.I.S.P.P., Forli.

SERNA J.L. 1991 : La industria lítica de la Cova del Xorret (Crevillent, Alicante). PLAV-Saguntum, 24, pp. 9-21. 
SOLER N. et J. MAROTO 1996 - Les grottes du Reclau (Serinyà, Catalogne) et le Gravettien du Méditerranéen Ibérique, The Upper Palaeolithique. The late Aurignacian. The Origin of the Gravetian, Coloquia, 6, XIII Congrès de I’U.I.S.P.P., Forli. pp. 295-312.

SOLER N. et GARCIA L. 1995 - Un probable xiulet paleolític a Davant Pau (Serinyà, el Pla de l'Estany), Cultura i Medi de la Prehistòria a l'Edat Mitjana, Puigcerdà, pp. 195-206.

SOLER N. et MAROTO J. 1987 a - L'estratigrafia de la cova de l'Arbreda (Serinyà, Girona), Cypsela, Vl, Girona, pp. 53-66.

SOLER N. et MAROTO J. 1987 b - Els nivells d'ocupació del Paleolític Superior a la cova de l'Arbreda (Serinyà, Girona), Cypsela, VI, Girona, pp. 221-228.

SOLER N. 1980 - El jaciment prehistòric del "Roc de la Melca"i la seva cronologia, Sant Aniol de Finestres (Girona), Annals de l'Institut d'Estudis Gironins, XXV, 1, Girona, pp. 43-58.

SOLER N. 1986 - El Paleolític Superior de Davant Pau (Serinyà), Quadems del Centre d'Estudis Comarcals de Banyoles, 1985, pp. 85-110.

SOLER N. 1986 - Les indústries del Paleolític Superior en el Nord de Catalunya. Tesi doctoral, Universitat de Barcelona.

SOLER N. 1994 - "Le Solutréen en Catalogne". Le Solutréen en Péninsule ibérique, Musée Départamental de Solutré, pp. 31-38.
UTRILLA P. y DOMINGO R. 2001/02 - "Excavaciones en el Arenal de Fonseca (Landruñán, Teruel)". Salduie, 2, pp. 337-354.

VILA A. 1987 - L'assentament paleolític del Castell (Vilanova De Sau), Cypsela, Girona, pp. 111-123.

VILLAVERDE V. 1979 - El Solutrense en el País Valenciano. Estado actual de su conocimiento. Saguntum- PLAV, 14, pp. 9-31.

VILLAVERDE V. (2001): El Paleolítico superior: El tiempo de los cromañones. Periodización y características. De Neandertales a Cromañones. Los inicios del poblamiento humano en el País Valenciano (ed. V. Villaverde). Universitat de València. pp. 177-218.

VILLAVERDE V., AURA J.E. et BARTON C.M. 1998 " The Upper Paleolithic in Mediterranean Spain: A Review of Current Evidence ". Journal of World Prehistory, 12 (2), pp. 121-198.

VILLAVERDE V. et ROMAN D. 2004 - "Avance al estudio de los niveles gravetienses de la Cova de les Cendres. Resultados de la excavación del sondeo (cuadros A/B/C-17) y su valoración en el contexto del Gravetiense mediterráneo ibérico". Archivo de Prehistoria Levantina XXV, pp. 19-59.

ZILHÃO J. 2003 - Vers une chronologie plus fine du cycle ancien de l'art paléolithique de la Côa: quelques hyppothèses de travail. El Arte Prehistóriuco desde los inicios del siglo XXI (R. de Balbín y P. Bueno, edts.). pp. 75-87. 